\title{
Evidencia de la conectividad ecológica del cerro Tacarcuna, expresada en la presencia y distribución de mamíferos terrestres neotropicales
}

\section{Evidence of ecological connectivity of cerro Tacarcuna, expressed in the presence and distribution of neotropical terrestrial mammals}

\author{
Eric Yair Cuesta-Ríos*
}

\section{RESUMEN}

El presente documento exhibe una serie de anotaciones ecológicas que evidencian la conectividad ecológica del cerro Tacarcuna y que se expresa en la presencia y distribución de un grupo de mamíferos que se distribuyen desde Norteamérica hasta Suramérica. Para lograr asociar la conectividad de Tacarcuna con la presencia y distribución de la mastofauna, se caracterizó ecológicamente la fauna de mamíferos, a partir de observaciones de campo enfatizadas en puntos de muestreo específicos por grupo taxonómico, aplicando la metodología de Muestreos Ecológicos Rápidos y la aplicación de entrevistas informales; posteriormente se recurrió a la revisión de literatura especializada para determinar la distribución de las especies. La mastofauna estuvo representada por 17 especies, todas distribuidas dentro del corredor ecológico EE.UU.-Argentina, correspondientes a los órdenes; Rodentia, Lagomorpha, Artiodactyla, Cingulata, Primates, CarnIvora y Perissodactyla, donde los roedores, carnívoros y primates fueron los mejor representados. Se reconoció la fragmentación del bosque como el factor que limitó, en gran parte, la presencia de las especies, pero paradójicamente favoreció la confluencia de otras como Cuniculus paca, Akodon sp., Sciurus granatensis y Sciurus depeii. Se registra por primera vez la especie S. deppei, para Colombia. Asimismo se destaca la presencia de Tapirus bairdii, que junto con Panthera onca y Puma concolor, son de las especies más amenazadas de Colombia, asumiendo la presencia de numerosas huellas y como indicador de la abundancia de sus poblaciones, lo que sugiere un plan de manejo para el área, con el objetivo de evitar la pérdida de biodiversidad.

Palabras clave; Cerro de Tacarcuna; Conectividad ecológica; Mamíferos terrestres neotropicales; Sciurus depeii; Tapirus bairdii.

\begin{abstract}
The present document shows a series of ecological annotations that demonstrate the ecological connectivity of the cerro Tacarcuna and that one expresses in the presence and distribution of a group of mammals that distribute from North America up to South America. To manage to associate Tacarcuna 's connectivity with the presence and distribution of the mastofauna, the fauna of mammals was characterized ecologically, from field observations emphasized specific points of sampling by group taxonómico, applying the methodology of Ecological Rapid Samplings and the application of informal interviews, later one resorted to the review of specialized literature to determine the distribution of the species. The mastofauna was represented by 17 species, all distributed inside the ecological corridor USA-Argentina, correspondents to the orders; Rodentia,
\end{abstract}

* Corporación Autónoma Regional para el Desarrollo Sostenible del Chocó (CODECHOCO), Grupo de Investigación Conocimiento, Manejo y Conservación de los Ecosistemas del Chocó Biogeográfico, Quibdo, Colombia. e-mail: cuestarios@gmail.com Recibido: 14 de junio de 2011 Aceptado: 20 de septiembre de 2011 


\section{Bioetnia Volumen 8 No 2 (julio-diciembre), 2011}

Lagomorpha, Artiodactyla, Cingulata, Primates, Carnivorous and Perissodactyla, where the rodents, carnivorous and primates were them better represented. The fragmentation of the forest was recognized as the factor that limited, largely, the presence of the species, but paradoxically the confluence favored of others as Cuniculus paca, Akodon sp., Sciurus granatensis and Sciurus depeii. There is registered by the first time the species $S$. deppei, for Colombia. Likewise is outlined the presence of Tapirus bairdii, which together with Panthera onca and Puma concolor, they are of the species most threatened with Colombia, assuming the presence of numerous fingerprints and as indicator of the abundance of his populations, which suggests a plan of managing for the area, with the aim to avoid the loss of biodiversity.

Keywords: Cerro de Tacarcuna; Ecological connectivity; Neotropical terrestrial mammals; Sciurus depeii; Tapirus bairdii.

\section{INTRODUCCIÓN}

Existen diversos factores que han contribuido a la colonización de muchas especies en diversos ecosistemas, entre los que están los corredores ecológicos que han favorecido al mantenimiento y recambios genéticos, la propagación de las especies, favorecimiento de las migraciones y conexión de una variada gama de ecosistemas, permitiendo la sostenibilidad de la biodiversidad existente.

Un corredor ecológico es aquel ámbito territorial cuya función primordial es la de conectar dos o más sectores con características ambientales similares, de forma que resulte transitable y sirva como conducto a los desplazamientos de la biota (Gurrutxaga y Lozano 2008). De esta manera, los corredores ecológicos corresponden con sectores de la matriz territorial que presentan un especial interés para mantener la conectividad ecológica, entendida como la capacidad del territorio para permitir los desplazamientos de las especies silvestres entre las teselas con recursos, donde la conectividad es un elemento fundamental del paisaje (Taylor et al. 1993) y así, una de las estrategias para mitigar los efectos de la fragmentación sobre los ecosistemas consiste en mantener o restablecer los vínculos en el paisaje (Noss 1991).

La conectividad también describe cómo los arreglos espaciales y la calidad de elementos en el paisaje afectan el desplazamiento de organismos entre parcelas de hábitats (Benett 2004). En términos de manejo, la conectividad encuentra su manifestación en los corredores biológicos (Worboys 2010); sin embargo, los corredores pueden representar tanto una oportunidad de desplazamiento para los organismos, como una barrera, dependiendo del tipo de cobertura del corredor y el organismo particular (Anderson y Jenkins 2006, Hilty et al. 2006). Es por esta razón, que tanto los eslabones de ecosistemas naturales, como la matriz circundante, juegan un papel importante en la conectividad ecológica (Schelhas y Greenberg 1996, Benett 2004).

En el nuevo mundo, factores como la consolidación del istmo de Panamá y consigo el afianzamiento del corredor terrestre del mismo, propiciaron la formación de mosaicos paisajísticos y elementos biológicos propios que le distinguen de otras regiones geográficas, por su diversidad, abundancia y distribución. Claro ejemplo de esto es el cerró de Tacarcuna, que contiene una biota única, cada una con sus particularidades, donde factores como la humedad relativa, las precipitaciones, la temperatura y la topografía de cada sitio en particular, juegan un papel fundamental en el establecimiento y mantenimiento de cada uno de los elementos bióticos que allí convergen, lo que ratifica que esa zona ha sido un corredor biológico entre las Américas. Asimismo, los bosques de este lugar han sido reconocidos por su elevadísima biodiversidad y por presentar gran número de endemismos producto de sus características, que permitieron el ensamblaje de muchos grupos biológicos que encontraron en Tacarcuna condiciones óptimas para su establecimiento.

Los mamíferos por su alta movilidad han sido un grupo que se ha expandido notablemente en América, gracias a su capacidad adaptativa a diversos ecosistemas, entre estos Tacarcuna y el corredor biológico que interconecta a las Américas, permitiendo que exista un flujo genético y consigo especiaciones que han aumentado la diversidad y distribución de estos grupos, que además cumplen actividades ecológicas como la dispersión de semillas, favoreciendo de esta manera las dinámicas de regeneración natural del bosque, y por consiguiente, el mantenimiento de las comunidades vegetales y su entorno. A partir de las razones aquí expuestas se presenta un documento que evidencia la conectividad ecológica del cerro Tacarcuna, expresada en la presencia y distribución de mamíferos terrestres neotropicales.

Las anteriores razones, aunadas al hecho de que este cerro es un extenso corredor, implica la amplia distribución de las especies que habitan esta ecorregión, pues no existen barreras geográficas que impidan su paso a lo largo del complejo. Además, el alto número de especies endémicas, así como especies migratorias, permiten que este lugar represente un ecosistema con un gran valor potencial para la conservación, lo que sugiere un plan de manejo del lugar con el objetivo de protegerlo de la pérdida de biodiversidad por fragmentación o por explotación del recurso.

Las apreciaciones anotadas anteriormente recaen sobre muchos argumentos del orden científico y académico; por esta razón, entidades como el Instituto de Investigaciones Ambientales del Pacífico, la Corporación Autónoma Regional para el Desarrollo Sostenible del Chocó, la Universidad 
Tecnológica del Chocó y diversos consejos comunitarios, están aunando esfuerzos en su aspiración por el conocimiento y valoración de la biodiversidad, para atender mejor estos recursos en beneficio de las poblaciones humanas. Por tanto, el conocimiento, manejo y aprovechamiento de la biodiversidad se expande y diversifica, en busca de políticas, enfoques y técnicas acordes con las necesidades y escenarios de la región. En el marco de esta expresión, se caracterizó ecológicamente la mastofauna terrestre del cerro de
Tacarcuna, como una estrategia de proyección que sirva como herramienta para el establecimiento de una figura de conservación en el Chocó biogeográfico.

Descripción del área de estudio. El estudio se realizó en el cerró Tacarcuna, jurisdicción del municipio de Unguía, Chocó, en un rango altitudinal que comprendió elevaciones de 1000-1800 mnsm (Figura 1). El lugar muestreado presentó una vegetación bastante diferenciada de las zonas bajas que lo circundan, las cuales han sido transformadas a causa

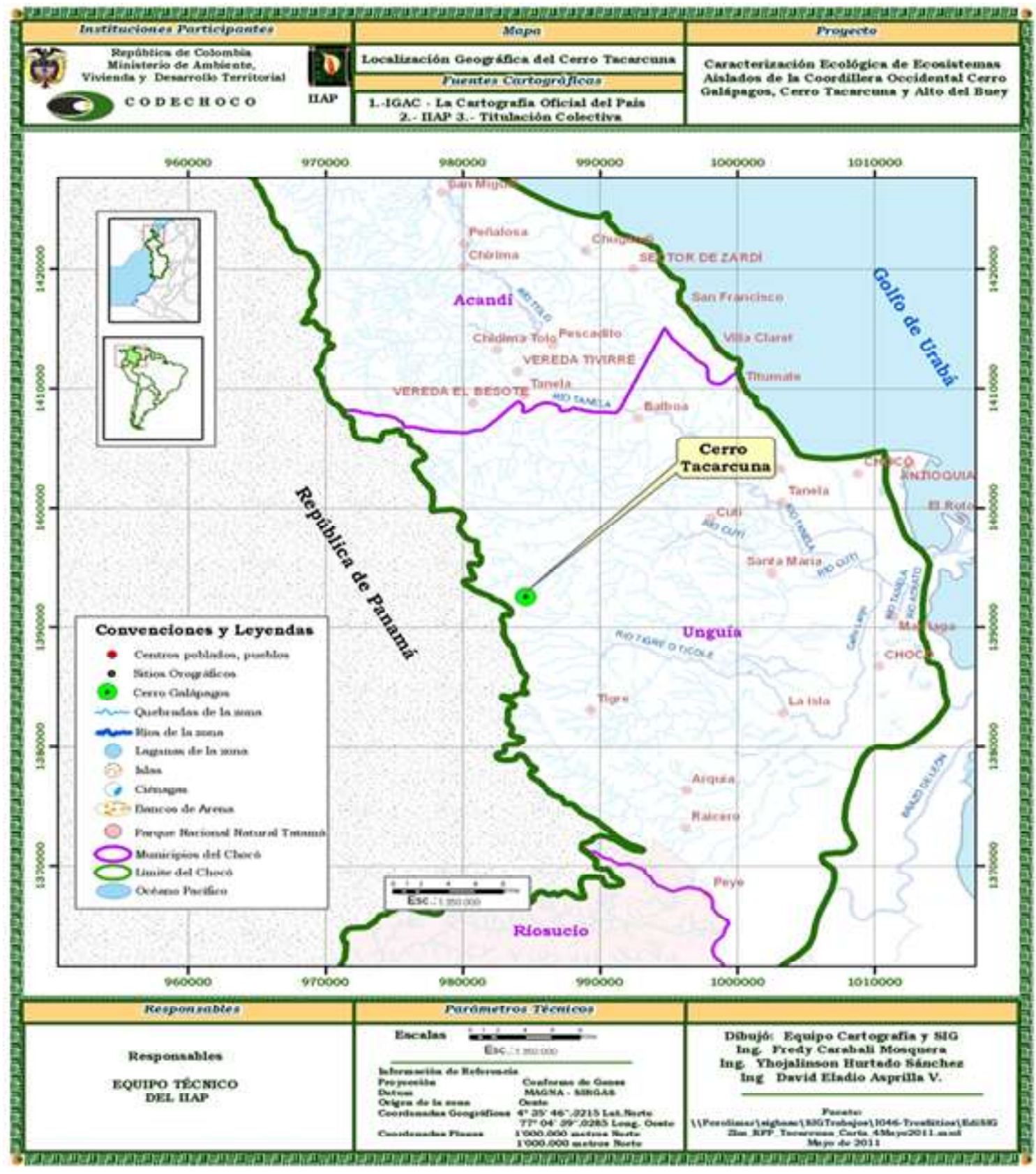

Figura 1. Ubicación geográfica del cerro de Tacarcuna 


\section{Bioetnia Volumen 8 No 2 (julio-diciembre), 2011}

de la expansión de la frontera agrícola y la ganadería; sin embargo, las partes altas del cerro están cubiertas con un bosque espeso, algunas extensiones de selva húmeda hacia las partes más altas y mínimas fracciones de bosques fragmentadas con la presencia de pequeños cultivos agrícolas. En la zona se encontraron bosques representados por Anacardium excelsum, Brosimun utile y bosques de Pseudolmedia laeviagata, y en menor proporción selvas con Prioria copaifera y Carapa guianensis (Orjuela-R et al 2004). También predominan especies listadas en los libros rojos de Colombia como es el caso del roble (Quercus humboltdii); la zona exhibe una alta diversidad de otros grupos biológicos como aves, anfibios y mariposas siendo la misma el nacimiento de un gran número de fuentes lóticas que drenan sus aguas al río Atrato.

\section{MÉTODOS}

Para la evaluación de la mastofauna se seleccionaron múltiples sitios de trabajo atendiendo los requerimientos ecológicos de cada grupo faunístico, partiendo exclusivamente de la metodología aplicada para estos taxones. Se realizaron observaciones de campo enfatizadas en puntos de muestreo específicos por grupo taxonómico, aplicando la metodología de Muestreos Ecológicos Rápidos «MER» (Noss 1990). La aplicación del método permitió levantar información esencial en un corto tiempo en áreas previamente seleccionadas por su calidad ambiental y su potencial de biodiversidad o representatividad.

Se utilizó la combinación de métodos indirectos y directos según lo propuesto por Tirira (1999).

Recopilación de información secundaria. Se realizó por medio de entrevistas informales a personas clave (guías conocedores de fauna de la localidad), donde se indagó sobre la oferta y existencia de mamíferos en el lugar, información que permitió la recopilación de aspectos ecológicos de las comunidades animales que allí habitan.

Complementario a esto se recurrió a la revisión de literatura especializada, con el fin de determinar la distribución de las especies dentro de la cordillera de los Andes y mostrar de esta manera la importancia de Tacarcuna como punto de conexión y área puente de muchas especies que han tenido a través de la historia un paso obligado por este corredor desde Norte América hacia Sur América y viceversa.

Búsqueda e identificación de huellas y otros rastros. Se realizaron recorridos a lo largo de transeptos, aleatoriamente determinados en campo. Esta técnica buscó identificar huellas (pisadas) y otros rastros que revelaran la presencia de especies de mamíferos. Dentro de otros rastros, se incluyó la búsqueda de madrigueras, comederos, restos óseos, heces fecales, marcas de orina, así como la identificación de vocalizaciones.

\section{RESULTADOS Y DISCUSIÓN}

La mastofauna de Tacarcuna estuvo representada en 17 especies agrupadas en 11 familias y 15 géneros correspondientes a los taxones Rodentia, Lagomorpha, Artiodactyla, Cingulata, Primates, Carnivora y Perissodactyla, donde los roedores, carnívoros y primates fueron los mejor representados con cuatro especies cada uno (Tabla 1).

El registro a nivel específico de estos grupos, posiblemente obedece a factores propiciados por la diversificación ecosistémica y biológica de Tacarcuna con su posición geográfica de conexión y área puente de Suramérica con el Caribe, Centroamérica y el Pacífico; la formación de grandes corredores de intercambio biológico en la dirección nortesur y viceversa, a lo largo de la cordillera de los Andes desde los Estados Unidos hasta la Patagonia, influido por la conectividad entre paisajes, ecosistemas y hábitat, naturales o modificados, asegurando el mantenimiento de la diversidad biológica, procesos ecológicos y evolutivos, lo que sugiere la búsqueda de medidas que permitan la conservación de este lugar que fue, ha sido y será el paso obligado de un sinnúmero de especies animales entre las Américas, garantizando el flujo genético y la existencia de estas especies en las selvas tropicales de América.

Muchas de las taxa aquí documentadas, representan grupos netamente norteños en su origen, tales como los carnívoros y roedores, que en esta investigación fueron dos de los grupos más abundantes. Según Alberico (1993), muchos de estos grupos de animales pudieron aprovechar la conexión terrestre entre las serranías costeras y la cordillera Occidental para expandir su rango de distribución en el nuevo continente, logrando diversificarse en nuevos géneros y especies en América del Sur.

A la vez, casi la totalidad de la fauna de las selvas húmedas en el noroeste del continente sur pudo colonizar los bosques similares que se habían formado en Centroamérica, proporcionando un recambio específico que se ratifica en la distribución de muchas de estas especies, que para el caso del Chocó se encuentran desde la serranía del Darién hasta la del Baudó, siendo consideradas de amplia distribución a lo largo de la cordillera Occidental, porque sus distribuciones son mayores a $50.000 \mathrm{~km}$ (Terborgh y Winter 1983); gracias a sus requerimientos nutricionales y de hábitat realizan grandes desplazamientos y han encontrado en estos espacios las garantías de hábitat y alimento.

$\mathrm{Al}$ analizar la presencia y representatividad de roedores, carnívoros y primates en Tacarcuna, se puede inferir que este hecho se debe quizás a la composición florística de la zona que constituye un mosaico microhabitacional favorable para el desarrollo no solo de estos grupos sino de otros mamíferos que son importantes en el recambio y dinámica poblacional del entorno. 
Tabla 1

Listado y categoría de amenaza de mamíferos presentes en el cerro de Tacarcuna, Chocó, Colombia

\begin{tabular}{|c|c|c|c|c|c|c|c|}
\hline Orden & Familia & Especie & $\begin{array}{l}\text { Nombre } \\
\text { local }\end{array}$ & $\begin{array}{l}\text { Regi } \\
\text { Dir }\end{array}$ & & EC & DG \\
\hline Rodentia & $\begin{array}{l}\text { Cuniculidae } \\
\text { Sciuridae } \\
\text { Echimyidae }\end{array}$ & $\begin{array}{l}\text { Cuniculus paca } \\
\text { Sciurus granatensis } \\
\text { Sciurus deppei } \\
\text { Proechimys s } \\
\text { emispinosus }\end{array}$ & \begin{tabular}{|l|} 
Guagua \\
Ardilla roja \\
Ardilla pequeña \\
$\begin{array}{l}\text { Ratón de } \\
\text { monte }\end{array}$ \\
\end{tabular} & $\begin{array}{l}x \\
x \\
\end{array}$ & $x$ & & $\begin{array}{l}\text { Méx hasta Par y el sur de } \\
\text { Bra } \\
\text { Norte de C. Rica hasta } \\
\text { Ecu, norte de Ven y } \\
\text { Centro y occidente de Col } \\
\text { Centro América } \\
\text { Desconocida }\end{array}$ \\
\hline Lagomorpha & Leporidae & Sy/vilagus brasiliensis & Conejo burro & $x$ & & & Méx hasta el norte de Arg \\
\hline Artiodactyla & Tayassuidae & $\begin{array}{l}\text { Tayassu pecari } \\
\text { Pecari tajacu }\end{array}$ & \begin{tabular}{|l|} 
Mana o tatabro \\
Sainó de collar
\end{tabular} & & $\mathrm{X}$ & $\mathrm{VU}$ & $\begin{array}{l}\text { Méx hasta el oeste de Ecu } \\
\text { y norte de Arg } \\
\text { Sudoeste de EE.UU hasta } \\
\text { el norte de Arg }\end{array}$ \\
\hline Cingulata & Dasypodidae & Dasypus novemcinctus & Armadillo & $x$ & & & $\begin{array}{l}\text { Sur de los EE. UU hasta el } \\
\text { Norte de Arg }\end{array}$ \\
\hline Primates & Callitrichidae & $\begin{array}{l}\text { Sanguinus geoffroyi } \\
\text { Sanguinus oedipus } \\
\text { Ateles fusciceps } \\
\text { Cebus capucinus }\end{array}$ & $\begin{array}{l}\text { Michichi } \\
\text { Michichi } \\
\text { Mica negra } \\
\text { Cariblanco }\end{array}$ & $x$ & & $\mathrm{VU}$ & $\begin{array}{l}\text { Endémica del complejo } \\
\text { Chocó Biogeográfico: Col - } \\
\text { Pan } \\
\text { Endémica de Colombia: } \\
\text { noroeste de Col } \\
\text { América Central y del Sur: } \\
\text { Ecu, Ven, Col, Par, Arg, } \\
\text { Bra, Pan, C. Rica y Per } \\
\text { América Central y en una } \\
\text { pequeña porción de Sur } \\
\text { América: Col y Ecu }\end{array}$ \\
\hline Carnivora & Procyonidae & \begin{tabular}{|l|} 
Potos flavus \\
Nasua narica \\
\\
Panthera onca \\
Puma concolor \\
\end{tabular} & \begin{tabular}{|l|} 
Perro de monte \\
Cuzumbo \\
\\
Tigre \\
León \\
\end{tabular} & $x$ & $\begin{array}{l}X \\
X \\
\end{array}$ & $\begin{array}{l}\text { VU } \\
\text { VU }\end{array}$ & $\begin{array}{l}\text { Sur y Centro América: } \\
\text { Desde el sur de Méx a Bol } \\
\text { y Mato Grosso en Bra, Col, } \\
\text { Ven, Guy, Suri, Ecu, Per } \\
\text { Suroeste de E.E.U.U. } \\
\text { hasta el este de Pan y el } \\
\text { norte de Col } \\
\text { Méx hasta Arg } \\
\text { EE. UU hasta Arg }\end{array}$ \\
\hline $\begin{array}{l}\text { Perisso- } \\
\text { dactyla }\end{array}$ & Tapiridae & Tapirus bairdii & Danta & & $\mathrm{X}$ & $\mathrm{CR}$ & $\begin{array}{l}\text { México hasta noroeste de } \\
\text { Ecu y el norte de Col. }\end{array}$ \\
\hline
\end{tabular}




\section{Bioetnia Volumen 8 No 2 (julio-diciembre), 2011}

En el caso de los primates y roedores que se observaron con mucha frecuencia entre los $1200 \mathrm{msnm}$ y $1600 \mathrm{msnm}$, su presencia puede estar enmarcada dentro de las características de la cobertura vegetal del bosque, que a pesar de presentar zonas fragmentadas que suelen emplearse para el establecimiento de cultivos agrícolas, proveen a los roedores y primates una gran oferta trófica que de buena manera puede estar influido directamente con una mayor confluencia de especies en busca de alimento; sin duda los roedores como Cuniculus paca, Proechimys semispinosus, Sciurus granatensis y Sciurus depeii, sacan mayor provecho de esto, porque la fragmentación del hábitat con cultivos les facilita la consecución de alimento.

A pesar de la presencia de zonas fragmentadas, el hábitat suele ser continuo, lo que consiente la formación de una conectividad biológica que en los procesos de movilidad, alimentación y reproducción, favorece en gran medida la presencia de diversos primates que se observaron notablemente desplazándose por la cobertura boscosa y en algunas zonas fragmentadas alimentándose y realizando aspectos sociales como el acicalamiento y amamantamiento de crías.

Paradójicamente, la principal amenaza para la supervivencia de los primates y de muchos otros vertebrados es la pérdida y fragmentación del su hábitat. Fahrig (1998; 2003) manifiesta que a medida que el grado de fragmentación aumenta, se reduce la cantidad total de hábitat disponible, se incrementa el número de parches de hábitat, disminuye su tamaño medio, aumenta su aislamiento y se reduce su calidad.

En los primates, que presentan hábitos arbóreos, este fenómeno ocasiona cambios en la estructura demográfica y poblacional, estimulando aislamiento de las unidades sociales y de los individuos, lo que los hace más susceptibles y altamente vulnerables a la extinción local; probablemente este grupo ha adoptado estrategias como la distribución y uso de hábitat, que son mecanismos que posiblemente le han permitido garantizar su supervivencia y hacerle frente al problema de la fragmentación y a patrones etológicos como la territorialidad que se presenta en la mayor parte de los primates, porque reducen la competencia intra e interespecífica permitiendo la jerarquización dentro del bosque y adoptando sus conductas ecológicas a la oferta y disponibilidad de hábitat y alimento.

Fleagle y Mittermeier (1980) y Tomblin y Cranford (1994), manifiestan que las diferencias entre comportamiento alimenticio, dieta y uso de hábitat arbóreo juegan un papel importante en la separación de nichos ecológicos; en este caso los primates pueden convivir en un mismo hábitat utilizando diferentes parámetros ecológicos del mismo a fin de evitar competencia.

Al relacionar la ecología y zoogeografía de la primatofauna de Tacarcuna se puede apreciar que existe una similitud del
$80 \%$ entre las especies del noroeste de Colombia con Panamá, factor asociado a la extensión del bosque lluvioso de Centroamérica hacia el occidente colombiano que se integra al corredor biológico del Chocó biogeográfico.

En relación con el orden Carnivora se registraron las familias Felidae, Procyonidae, que se limitan conforme a los requerimientos habitacionales y tróficos. La presencia de los felinos en Tacarcuna corresponde quizás a la alta oferta alimenticia que les permite satisfacer sus necesidades y requerimientos alimenticios, dado que en este complejo montañoso se alberga un sinnúmero de presas potenciales del puma y jaguar. Aunque no fue posible el encuentro visual de estos felinos por los hábitos silvícolas y estrategias demográficas que poseen, se puede ratificar a groso modo la presencia de estas especies en el ecosistema, gracias al gran número de huellas y heces fecales hallados, que permiten corroborar la presencia de estos felinos en Tacarcuna.

La presencia del jaguar (Panthera onca) y el puma (Puma concolor) evidentemente se puede ver enmarcada bajo la presencia de presas potenciales de estos felinos que quizás correspondan a mamíferos de porte mediano como los puercos de monte (Tayassu pecari, Pecari tajacu) y la guagua $(C$. paca), y animales de porte pequeño como el armadillo (Dasypus novemcinctus) e incluso sobre otros carnívoros como Nasua narica y Potos flavus, de lo que se infiere que los carnívoros son presa ocasional, aunque no rara del puma.

En estudios similares en otras latitudes continentales, se ha reportado que el puma y el jaguar depredan sobre felinos más pequeños, zorros, prociónidos, osos negros y hasta sobre otros pumas (Yañez et al. 1986, Maehr et al. 1990). De igual manera, Leyequién y Balvanera (2007), Núñez et al. (2002), Amin (2004), Rosas-Rosas et al. (2008), consideraron como presas potenciales de estos felinos a la guagua, pecarí de collar, coatí y armadillo, grupos biológicos con abundancias relativas significativas en el ecosistema.

En un término más amplio, el papel ecológico de los felinos como predadores, en este caso el puma y jaguar, hace que usualmente tengan unos rangos geográficos muy amplios en una variedad de hábitats, lo que quizás les permitió desplazarse desde Norteamérica, encontrando refugio en la geografía suramericana, ampliando así su distribución geográfica en las selvas neotropicales, tal vez propiciadas por un sinnúmero de características biofísicas, a lo largo de un inmenso espacio de conectividad ecológica llamado Tacarcuna, que presenta atributos de composición, estructura y fusión que favorecen el desarrollo de muchas actividades etológicas de estos y de otros grupos biológicos, demostrando de esta manera la importancia de este lugar como refugio y ruta de migración ecológica y genética, para un inmenso contingente faunístico que se encuentra en el vasto territorio americano.

Siguiendo con los carnívoros, existen otras especies que 
sus distribuciones también se han visto favorecidas por los canales de conexión biológica, porque se les encuentra desde los Estados Unidos hasta algunas zonas de Suramérica como $N$. narica y $P$. flavus, que con frecuencia se observaron alimentándose sobre todo de material de origen vegetal (semillas, frutos y flores), elementos biológicos muy frecuentes en el ecosistema, que brinda una oferta trófica variada, patrón notorio que se registró durante el estudio, donde se observó que ciertas plantas que habitan el Tacarcuna, fenológicamente se encontraban en período de floración y fructificación lo que también se constituye en un recurso alimenticio, teniendo en cuenta que estos elementos vegetales permiten la atracción de la entomofauna que también hacen parte de la diversidad trófica de estas especies.

Hubo otros grupos que a pesar de que en el ecosistema no fueron representativos en abundancia específica, si fueron importantes en la dinámica poblacional por ser elementos primordiales en las cadenas tróficas y flujo energético, como es el caso de los ungulados Tapirus terrestris, T. pecari y $P$. tajacu, cuya presencia se percató en la zona por las numerosas huellas y rastros encontrados en el ecosistema; es posible que al ser Tacarcuna un sitio poco perturbado y con vegetación secundaria, puede estar favoreciendo la presencia de especies de hábitos generalistas, como los Tayassuidos.

El zaíno de collar que entre los ungulados fue el más representativo, porque la mayor parte de las huellas correspondían a esta especie, se distribuye preferiblemente en zonas de bosque secundarios (Gonzales-Marín et al 2008), porque estos posiblemente le proporcionan una mayor diversidad de especies vegetales como los frutos, semillas y raíces. En la zona no fue posible observalos, pero sí se encontraron restos fósiles, huellas y heces fecales de grandes felinos compuesto por pelos, que al realizar el respectivo análisis correspondían a P. tajacu; esto puede indicar que se trata de elementos nutritivos en la dieta de felinos como el tigre y el jaguar.

Por los pocos rastros encontrados de $T$. pecari, se podría deducir que el estado de sus poblaciones fueron bajas con menores densidades poblacionales y una distribución restringida dentro del área; también se puede pensar en otros factores que explicarían la ausencia de rastros, como los grandes desplazamientos y el comportamiento impredecible de las manadas en busca de alimento y otros recursos (Kiltie y Terborgh 1983; Bodmer 1990; Fragoso 1998).

Se puede considerar como la anotación más importante de esta caracterización, el registro de la danta (T. bairdii), que es una especie de hábitos silvícolas y secretivos muy marcados, lo que limita su encuentro visual. La gran variedad de microhábitats que brinda Tacarcuna pudo ser el factor más importante para la permanencia de la danta, que es un mamífero que suele utilizar una variedad de hábitats en sus desplazamientos en búsqueda de recursos alimenticios; igual- mente contribuye con otros procesos ecológicos como la dispersión de semillas favoreciendo de esta manera las dinámicas de regeneración natural del bosque y por consiguiente, en el mantenimiento de las comunidades vegetales y su entorno.

Siendo $T$. bairdii una especie estrictamente vegetariana, el hecho de presentar una abundancia relativamente significativa puede estar asociado con la época en que fue realizado el estudio, que coincidió con el período de fructificación y floración de muchas especies vegetales, lo que explicaría el significativo número de rastros (heces, huellas y restos fósiles) que se encontraron en la zona; esto tal vez indica que virtualmente las poblaciones de este mamífero en el área son abundantes y saludables. Además, la presencia de la danta indica la calidad del hábitat, porque este animal es un buen indicador de la conectividad del paisaje, lo que resalta una vez más la importancia de la conservación de este puente de conectividad ecológica y genética de norte a sur y viceversa.

En término zoográfico la composición faunística del corredor biológico terrestre Panamá-Colombia, presenta una serie de especies favorecidas por las condiciones del mismo y por el gran intercambio faunístico entre América del Sur y del Norte. A raíz de la consolidación de este corredor terrestre, Ojasti (2000) señala que la fauna de esta región presenta su estampa propia y le distingue de otras regiones zoogeográficas, por la presencia de linajes antiguos y exclusivos o endémicos, así como de grupos procedentes de otras regiones, pero que caracterizan la fauna actual del neotrópico por su gran variedad, distribución o abundancia.

Igualmente manifiesta que en el intercambio faunístico entre América del Sur y del Norte, varios linajes de mamíferos del norte invadieron América del Sur: las musarañas, carnívoros, tapires y caballos, pecaríes, camélidos y venados, ardillas y conejos; asimismo grupos como los Didelfidos, armadillos, osos hormigueros, perezosos y algunos primates se han dispersado desde el sur hacia el Neártico, todos estos grupos muy variados y abundantes en las selvas suramericanas.

En términos ecológicos generales, el registro de 17 especies puede considerarse como representativo, gracias a la abundante oferta trófica en cada uno de los niveles energéticos y al rango altitudinal donde se encontraron, dado que las variaciones altitudinales jugaron un punto a favor; con respecto a la riqueza de los intervalos altitudinales cabe mencionar que los resultados encontrados representan una alta diversidad en altitudes intermedias (1000-1800 msnm). Clements (1916) señala que la composición de las especies y la estructura de una comunidad sufren cambios al tiempo que responden a gradientes ambientales, siendo mayores en estas zonas de transición entre elevaciones altas y bajas que se enriquecen con comunidades de estos estratos (Lomolino 2001), posiblemente guardando relación con las condiciones óptimas de la temperatura, de la cobertura y diversidad de 


\section{Bioetnia Volumen 8 No 2 (julio-diciembre), 2011}

vegetales.

Es cierto que la diversidad pudo haber sido mayor, pero factores como el cambio repentino de las variables climáticas y el corto tiempo de muestreo pudieron incidir directamente sobre la diversidad mastofaunística de Tarcarcuna; sin embargo, la colonización de estas especies encontradas en este tipo de bioma las constituye en elementos biológicos únicos e indispensables en los procesos ecológicos de esta importante y compleja zona orográfica persistente en el complejo Tacarcuna.

\section{LITERATURA CITADA}

Alberico, M. 1993. Los mamiferos de la planicie. p. 240-7. En: Leyva, P. (ed.) Colombia Pacifíco. Bogotá: Fondo FEN Colombia.

Anderson, A., C. N. Jenkins. 2006. Applying nature's design. Corridors as a strategy for biodiversity conservation. New York: University of Columbia Press. $\mathrm{X}+231 \mathrm{p}$.

Amín, M. 2004. Patrones de alimentación y disponibilidad de presas del jaguar (Panthera onca) y del puma (Puma concolor) en la reserva de la Biosfera Calakmul, Campeche, México. (Tesis de maestría). México: Instituto de Ecología, UNAM. 63 pp.

Bennett, A. F. 2004. Enlazando el paisaje: el papel de los corredores y la conectividad en la conservación de la vida silvestre. San José: Unión Mundial para la Conservación de la Naturaleza (UICN). 278 pp.

Bodmer, R. E. 1990. Responses of ungulates to seasonal inundations in the Amazonian floodplain. J Trop Ecol. 6: 191-201.

Clements, F. E. 1916. Plant succession: An analysis of the development of vegetation. Washington, DC: Carnegie Institute. $512 \mathrm{p}$.

Fahrig, L. 1998. When does fragmentation of breeding habitat affect population survival? Ecol Modelling. 105: 273-92.

Fahrig, L. 2003. Effects of habitat fragmentation on biodiversity. Ann Review Ecol Evol System. 34: 487-515.

Fleagle, J. G., R. A. Mitermeier. 1980. Locomotor behavior, body size, and comparative ecology of seven Surinam monkeys. Am J Phys Anthropol. 52: 301-14.

Fragoso, J. M. 1998. Home range and movement pattcrns of white-lipped pcccary (Tayassu pecari) herds in the northern Brazilian Amazon. Biotropica 30: 458-69.

González-Marín, R. M., S. Gallina., S. Mandujano, M. Weber. 2008. Densidad y distribución de ungulados silvestres en la reserva ecológica El Eden, Quintana Roo, México. Acta Zool Mex. (Nueva serie) 24 (1): 73-93.

Gurrutxaga, M., P. J. Lozano. 2008. Evidencias sobre la eficacia de los corredores ecológicos: ¿Solucionan la problemática de fragmentación de hábitats? Obser Med Amb.11: 171-83.

Hilty, J. A, W. Z. Lidicker, A. M. Merenlender. 2006. Corridor ecology: the science and practice of linking landscapes for biodiversity conservation. Washington, DC: Island Press. 323 pp.

Kiltie, R. A., J. Terborgh. 1983. Observations on the behavior of rain forest peccaries (Tayassu pecari) in Perú: Why do White-lipped peccaries form herds? Z. Tierpsychol 62: 214-7.

Leyequién, L., R. M. Balvanera. 2007. El jaguar en el este de la Huasteca Potosina. In: Ceballos, G., C. Chávez, R. List y H. Zarza (eds.). Conservación y manejo del jaguar en México. Estudio de caso y perspectivas. México, DF: CONABIO, WWF, Telcel, UNAM. p. 51-8.

Lomolino, M. V. 2001. Elevational gradients of species-density and prospective views. Elevational gradients in mammals: Special issue. Global Ecol Biogeogr. 10: 3-13.

Maehr, D. S., R. C. Belden, E. D. Land, L. Wilkins. 1990. Food habits of panthers in southwest Florida. J Wildlife Managem. 54: 420-3.

Noss, R. 1990. Indicators for monitoring biodiversity: A hierarchical approach. Conserv Biol. 4: 355-64.

Noss, R. F. 1991. Landscape connectivity: different functions at different scales. In: Hudson, E. (ed.). Landscape linkages and biodiversity. Washington, DC: Island Press: 27-39.

Núñez, R., B. Miller, F. Lindzey. 2000. Food habits of jaguars and pumas in Jalisco, México. J Zool. 252: 373-9.

Ojasti, J .2000. Manejo de fauna neotropical. En: F. Dallmeier (ed). SIMAB. Serie $\mathrm{N}^{\circ}$ 5. Washington, DC: Smithsonian Institution/MAB Program. $309 \mathrm{pp}$.

Orjuela-R., M.A., J.O. RangeL-CH. A. Garzón-C. 2004. Usos de las plantas en el Chocó biogeográfico. En: Rangel-Ch., J.O. (ed.). Colombia diversidad biótica, IV. El Chocó biogeográfico/costa pacífica. Bogotá: Instituto de Ciencias Naturales. p. 911-36.

Rosas-Rosas, O., L. Bender, R. Valdez. 2008. Jaguar and puma predation on cattle calves in northeastern Sonora, México. Rageland Ecol Manag. 61: 554-60.

Schelhas, J. R. Greenberg. 1996. The value of foret patches. In: Forest patches in tropical landscape. Washington, DC: Island Press. $426 \mathrm{pp}$.

Tirira, D. 1999. Técnicas de campo para el estudio de mamíferos silvestres. En: Tirira, D. (Ed.). Biología, sistemática y conservación de los mamiferos del Ecuador. Quito: SIMBIOE. Publicación Especial 1. pp. 93-125.

Taylor, P. D., L. Fahrig, K., Henein, G. Merriam. 1993. Connectivity is a vital element of landscape structure. Oikos 68: 571- 3.

Tomblin, D. C., J. A. Cranford. 1994. Ecological niche differences between Alouatta palliata and Cebus capucinus comparing feeding modes, branch use, and diet. Primates 35 (3): 265-74.

Worboys, G. L. 2010. The connectivity conservation imperative. In: Worboys, G.L., W. L. Francis, M. Lockwood (eds.). Connectivity conservation management. London: Earthscan. p. 3-21

Yañez, J., J. C. Cárdenas, P. Gezelle, F. M. Jaksic. 1986. Food habits of the southernmost mountain lions (Felis concolor) in South America: natural versus livestocked ranges. J Mammal. 67: 604-6. 